\title{
GENERALIZING THE PALEY-WIENER PERTURBATION THEORY FOR BANACH SPACES
}

\author{
Peter G. Casazza and Nigel J. Kalton
}

\begin{abstract}
We extend the Paley-Wiener pertubation theory to linear operators mapping a subspace of one Banach space into another Banach space.
\end{abstract}

\section{INTRODUCTION}

In his classical book on potential theory in 1877, Carl Von Neumann [13] introduced what we now call the Neumann series for a linear operator: If $X$ is a Banach space and $T: X \rightarrow X$ is a linear operator satisfying $\|I-T\|<1$, then $T$ is an onto isomorphism and $T^{-1}=\sum_{n=0}^{\infty}(I-T)^{n}$. Special cases of this result were rediscovered by Paley and Wiener in 1934 [10] and in 1940 by Boas [3]. After further generalizations by Pollard [11] and Sz. Nagy [9], Hilding [6] gave the most general form: If $X$ is a Banach space, and $T: X \rightarrow X$ is a linear operator, $\lambda \in[0,1)$, and for all $x \in X,\|(I-T) x\| \leq \lambda(\|x\|+\|T x\|)$, then $T$ is an onto isomorphism. We will investigate the more general setting where $Y$ is a subspace of a Banach space $X, Z$ is a Banach space and $S, T: Y \rightarrow Z$ are linear operators satisfying for all $x \in X$, the inequality, $\|S x-T x\| \leq \lambda_{1}\|S x\|+\lambda_{2}\|T x\|$, where $\lambda_{1}, \lambda_{2} \in[0,1)$. In this case, properties of $S$ will carry over to $T$. This includes being one-to-one, onto, closed, open, having dense range, being a quotient map and most importantly, being a Fredholm operator (and the Fredholm index is maintained). A special case of this result is a generalization of the theorem of Neumann: If $Y$ is a subspace of a Banach space $X$ and $T: Y \rightarrow X$ is a linear operator with $\left\|\left.(I-T)\right|_{Y}\right\|<1$, then $Y$ and $T Y$ have the same codimension in $X$.

The first author was supported by NSF-DMS 9201357, the Danish Natural Science Research Council, grant no. 9401598, and grants from the University of Missouri Research Board, and the University of Missouri Research Council. The second author was supported by NSF-DMS 95000125. AMS Subject Classification: 46B03, 46B20. Key Words and Phrases: Paley-Wiener Perturbation Theory, spectrum, approximate fixed points. 


\section{The Basic Inequalities}

We will first develop the basic inequalities needed throughout the paper. We will always assume that $\lambda_{1}, \lambda_{2}$ are real numbers with $\lambda_{1}, \lambda_{2} \in[0,1)$.

Basic Inequality. If $x, y$ are elements of a Banach space $X$ satisfying,

$$
\|x-y\| \leq \lambda_{1}\|x\|+\lambda_{2}\|y\|
$$

then,

$$
\frac{1-\lambda_{2}}{1+\lambda_{1}}\|y\| \leq\|x\| \leq \frac{1+\lambda_{2}}{1-\lambda_{1}}\|y\| .
$$

Proof. With $x, y$ as above,

$$
\|x\| \leq\|x-y\|+\|y\| \leq \lambda_{1}\|x\|+\lambda_{2}\|y\|+\|y\|
$$

It follows that,

$$
\|x\| \leq \frac{1+\lambda_{2}}{1-\lambda_{1}}\|y\| .
$$

Switching the roles of $x$ and $y$ above completes the inequality.

We will be working in the case where $Y, Z$ are subspaces of a Banach space $X$ and $T: Y \rightarrow Z$ is a bounded linear operator. Letting $y=T x$ in (1), we have for all $x \in Y$,

$$
\|(I-T) x\| \leq \lambda_{1}\|x\|+\lambda_{2}\|T x\|
$$

It follows from our basic inequality that $T$ is an isomorphism of $Y$ into $X$. We now show that if $T$ satisfies (3), then so do certain operators obtained from $T$.

Proposition 1. Let $Y, Z$ be subspaces of a Banach space $X$ and $T: Y \rightarrow Z$ be a surjective linear operator satisfying for all $x \in Y$,

$$
\|(I-T) x\| \leq \lambda_{1}\|x\|+\lambda_{2}\|T x\|
$$

Then

(1) $T^{-1}$ satisfies for all $x \in Z$,

$$
\left\|\left(I-T^{-1}\right) x\right\| \leq \lambda_{2}\|x\|+\lambda_{1}\left\|T^{-1} x\right\| .
$$


(2) For every $\alpha>0$, $\alpha T$ satisfies for all $x \in Y$,

$$
\|(I-\alpha T) x\| \leq \lambda_{1}^{\prime}\|x\|+\lambda_{2}^{\prime}\|\alpha T x\|
$$

with constants $\lambda_{1}^{\prime}=\max \left\{1-\alpha\left(1-\lambda_{1}, \lambda_{1}\right\}\right.$, and $\lambda_{2}^{\prime}=\max \left\{1-\frac{1+\lambda_{2}}{\alpha}, \lambda_{2}\right\}$.

(3) For every $0 \leq \alpha \leq 1$, the operator $T_{\alpha}=(1-\alpha) I+\alpha T$ satisfies for all $x \in Y$,

$$
\left\|\left(I-T_{\alpha}\right) x\right\| \leq \lambda_{1}^{\prime}\|x\|+\lambda_{2}^{\prime}\left\|T_{\alpha} x\right\|
$$

where $\lambda_{1}^{\prime}=\alpha \lambda_{1}+(1-\alpha) \lambda_{2}$, and $\lambda_{2}^{\prime}=\lambda_{2}$.

(4) For every $a<0$, the operator aI $-T$ is an isomorphism.

Proof. (1) If $z=T x$, then

$$
\left\|\left(I-T^{-1}\right) z\right\|_{Z \rightarrow Y}=\|T x-x\| \leq \lambda_{1}\|x\|+\lambda_{2}\|T x\|=\lambda_{1}\left\|T^{-1} z\right\|+\lambda_{2}\|z\| .
$$

(2) Although this can be done in one case, we will do it in two cases to identify the exact constants obtained in each case.

Case I. Assume $\alpha \leq 1$.

For any $x \in Y$,

$$
\begin{aligned}
\|(I-\alpha T) x\| & =\|(1-\alpha) I+\alpha(I-T) y\| \\
& \leq(1-\alpha)\|x\|+\lambda_{1} \alpha\|x\|+\lambda_{2} \alpha\|T x\| \\
& =\left[1-\alpha\left(1-\lambda_{1}\right)\right]\|x\|+\lambda_{2}\|\alpha T x\| .
\end{aligned}
$$

Case II. Assume $\alpha>1$.

For any $x \in Y$,

$$
\begin{aligned}
\|x-\alpha T x\| & \leq\|(I-T) x\|+(\alpha-1)\|T x\| \\
& \leq \lambda_{1}\|x\|+\lambda_{2}\|T x\|+(\alpha-1)\|T x\|=\lambda_{1}\|x\|+\frac{\lambda_{2}+\alpha-1}{\alpha}\|\alpha T x\| .
\end{aligned}
$$

(3) For any $x \in Y$,

$$
\begin{aligned}
\left\|\left(I-T_{\alpha}\right) x\right\| & =\alpha\|(I-T) x\| \leq \alpha \lambda_{1}\|x\|+\alpha \lambda_{2}\|T x\|=\alpha \lambda_{1}\|x\|+\lambda_{2}\|\alpha T x\| \\
& \leq \alpha \lambda_{1}\|x\|+\lambda_{2}\|\alpha T x+(1-\alpha) x\|+\lambda_{2}(1-\alpha)\|x\| \\
& =\left[\alpha \lambda_{1}+(1-\alpha) \lambda_{2}\right]\|x\|+\lambda_{2}\left\|T_{\alpha} x\right\| .
\end{aligned}
$$


(4) This is immediate from (3) and the observation,

$$
a I-T=-(|a|+1)\left[\left(1-\frac{1}{|a|+1}\right) I+\frac{1}{|a|+1} T\right]
$$

If we weaken inequality 3 , for example by letting $\lambda_{1}=1$, we lose our conclusion that $T$ is a bounded linear operator. For example, it is immediate that $T: \ell_{p} \rightarrow \ell_{p}$ given by $T\left(\left\{a_{i}\right\}\right)=\left\{i a_{i}\right\}$, satisfies $\|(I-T) x\| \leq\|T x\|$, for all $x \in \ell_{p}$. Also, $T=0$ satisfies inequality 3 with $\lambda_{1}=1$. The next proposition shows that this is essentially all that can go wrong with the weaker inequality 3 .

Proposition 2. Let $Y$ be a subspace of a Banach space $X$ and $T: Y \rightarrow X$ be a linear map.

(1) Suppose $T$ is bounded and there exists $\lambda \in[0,1)$ such that for all $x \in Y$,

$$
\|x-T x\| \leq \lambda\|x\|+\|T x\|
$$

Choose $\delta \in[\lambda, 1)$ so that $\|T\| \leq \frac{\delta-\lambda}{1-\delta}$. Then $T$ satisfies for all $x \in Y$,

$$
\|x-T x\| \leq \delta(\|x\|+\|T x\|)
$$

(2) If $T^{-1}$ is bounded on $T(Y)$, and $T$ satisfies for all $x \in Y$,

$$
\|x-T x\| \leq\|x\|+\lambda_{2}\|T x\|
$$

then

$$
\|x-T x\| \leq \delta(\|x\|+\|T x\|)
$$

where $\delta \in[\lambda, 1)$ is chosen so that $\left\|T^{-1}\right\| \geq \frac{1-\delta}{\delta-\lambda}>0$.

Proof. (1) Since $\|T x\| \leq \frac{\delta-\lambda}{1-\delta}\|x\|$, we see that $(1-\delta)\|T x\| \leq(\delta-\lambda)\|x\|$. Hence, $\lambda\|x\|+\|T x\| \leq \delta(\|x\|+\|T x\|)$. (2) follows similarly.

\section{An Application from Operator Theory}

We denote the unit sphere of a Banach space $X$ by $S_{X}=\{x \in X:\|x\|=1\}$. Let $\sigma(T)$ denote the spectrum of an operator $T: X \rightarrow X$ and $\pi(T)$ denote the approximate point spectrum of $T$. That is, $\lambda \in \sigma(T)$ if $T-\lambda I$ is not invertible, and $\lambda \in \pi(T)$ if there is a sequence $x_{n} \in S_{X}$ so that $\left\|(T-\lambda I) x_{n}\right\| \rightarrow 0$. In this terminology, proposition 7.9 in [7] states, 
Theorem (Kalton, Peck, Roberts). The complement of the spectrum of $T$ is a clopen (i.e. both closed and open) set in the complement of the approximate point spectrum of $T$, which contains the unbounded component.

Corollary 3. Let $X$ be a Banach space and $T: X \rightarrow X$ be an isomorphism of $X$ into $X$.

(1) If the operator $\alpha I-T$ is an isomorphism for all $\alpha>0$, then $T$ is onto.

(2) If the operator $\alpha I-T$ is an isomorphism for all $\alpha<0$, then $T$ is onto.

Proof. By our assumption in (1), $[0, \infty)$ is a subset of the unbounded component of $\pi(T)^{c}$ and hence is a subset of $\sigma(T)^{c}$. (2) follows similarly.

Some assumption on $T$ in Corollary 3 is necessary, since without it $T=0$ satisfies the hypotheses. In the complex case, the hypotheses in theorem 3 could be stated more generally as: (1) If for some complex unit $\left|\lambda_{0}\right|=1$, we have that $\alpha \lambda_{0} I-T$ is an isomorphism for all $\alpha>0$, then $T$ is onto. Similarly for (2).

The classical Borsuk-Ulam theorem asserts that any continuous map from an n-dimensional sphere to itself must either be onto, or have both fixed points and antipodal points. The Borsuk-Ulam theorem fails for infinite dimensional Banach spaces, in its exact form, even for linear isometries (just take the shift operator on a Hilbert space). However, there is an approximate version of this theorem. We say that a sequence of elements $\left\{x_{n}\right\}$ in $S_{X}$ is an approximate fixed point sequence for a mapping $f: S_{X} \rightarrow X$ if $\lim _{n \rightarrow \infty}\left\|x_{n}-f\left(x_{n}\right)\right\|=0$. It is an approximate antipodal sequence for $f$ if $\lim _{n \rightarrow \infty}\left\|-x_{n}-f\left(x_{n}\right)\right\|=0$. Benyamini and Sternfeld [2] have shown that every infinite dimensional Banach space $X$ has a Lipschitz map of the unit ball of $X$ into itself without approximate fixed points. If $X=\ell_{p}$, for $1<p<\infty$, this map automatically satisfies inequality 3. But the theorem of Kalton, Peck, and Roberts above does yield an approximate version of the BorsukUlam theorem for linear isometries.

Borsuk-Ulam Theorem for Linear Operators. If $X$ is a Banach space and $T: X \rightarrow X$ is an isometry, then either $T$ is onto, or $T$ has both an approximate fixed point sequence and an approximate antipodal sequence. Moreover, if $X$ is complex and $T$ is an isometry which is not onto, then the spectrum of $T$ contains the unit circle.

Proof. All of this is immediate from Corollary 3 except the last statement which only requires the observation that if $T$ is an isometry which is not onto, then for all complex numbers $|\lambda|=1$, the operator $\lambda T$ is not onto. By Corollary 3 , there is 
an $\alpha>0$ so that $\alpha I-\lambda T$ is not an isomorphism. It follows that $\alpha=1$, so $I-\lambda T$ is not an isomorphism, and hence $\bar{\lambda} I-T$ is not an isomorphism.

Isometries which are onto need not have approximate fixed points or approximate antipodal points. To see this, define $T: \ell_{p} \rightarrow \ell_{p}$ by:

$$
T\left(a_{1}, a_{2}, a_{3}, a_{4}, \ldots\right)=\left(a_{2},-a_{1}, a_{4},-a_{3}, \ldots\right)
$$

Then $T$ is an isometry of $\ell_{p}$ onto itself, but $T$ has no approximate fixed points or approximate antipodal points. Hence, $\alpha I-T$ is an onto isomorphism for all $\alpha$.

It is clear that any operator satisfying inequality (3) cannot have approximate fixed points or approximate antipodal points. One would hope that an operator which fails inequality (3) would need to have approximate fixed points or approximate antipodal points. It is easily checked that this is the case in a uniformly convex space. To see that this is not true in general, let $T\left(a_{1}, a_{2}\right)=\left(a_{2},-a_{1}\right)$ be considered as an operator on $\ell_{1}^{2}$.

The classical perturbation theorem of Hilding [6] now follows.

Hilding's Pertubation Theorem. If $X$ is a Banach space and $T: X \rightarrow X$ satisfies, for all $x \in X$,

$$
\|(I-T) x\| \leq \lambda_{1}\|x\|+\lambda_{2}\|T x\|
$$

for some $\lambda_{1}, \lambda_{2} \in[0,1)$, then $T$ is onto.

Proof. Proposition 1 (4) states that $T$ satisfies hypothesis 2 of Corollary 3.

\section{Generalizing Paley-Wiener}

Now we will extend the theory to operators between subspaces of Banach spaces. We start with an elementary observation.

Lemma 4. Let $X, Y$ be Banach spaces and $S, T: X \rightarrow Y$ be linear operators satisfying,

$$
\|S x-T x\| \leq \lambda_{1}\|S x\|+\lambda_{2}\|T x\|
$$

for all $x \in X$, and fixed $\lambda_{1}, \lambda_{2} \in[0,1)$. Then if $S$ has closed range (respectively, is one-to-one, has dense range, is an open map, is a quotient map, is an isomorphism) 
then $T$ has closed range (respectively, is one-to-one, has dense range, is an open map, is a quotient map, is an isomorphism).

Proof. Applying our Basic Inequality to $S x, T x$ we have:

$$
\frac{1-\lambda_{2}}{1+\lambda_{1}}\|T x\| \leq\|S x\| \leq \frac{1+\lambda_{2}}{1-\lambda_{1}}\|T x\| .
$$

It follows that $L: S(X) \rightarrow T(X)$ defined by, $L(S x)=T x$, is a well defined onto isomorphism, which therefore has a unique extension to an isomorphism of $\overline{S(X)}$ onto $\overline{T(X)}$. This is all that is needed for the proof of the theorem.

Now we want to prove a deeper generalization of the Paley-Wiener perturbation theory. We will need a result of Krein, Krasnoselskii, and Milman [8], which can also be found in Gokhberg and Krein [5] or Day [4].

Lemma 5. Let $E, F$ be subspaces of a Banach space $X$ with $\operatorname{dim} F<\infty$ and $\operatorname{dim} E>\operatorname{dim} F$. Then there exists an $0 \neq x \in S_{E}$ such that

$$
1=\|x\|=d(x, F) .
$$

To prove our main result, we need two lemmas. The first is actually a special case of the result.

Lemma 6. Let $Y$ be a subspace of a Banach space $X$, and $T: Y \rightarrow X$ a linear operator satisfying $\left\|\left.(I-T)\right|_{Y}\right\|<1$. Then for every subspace $W$ in $Y$, $\operatorname{codim}_{X} W \geq$ $\operatorname{codim}_{X} T(W)$.

Proof. Let $\lambda=\left\|\left.(I-T)\right|_{Y}\right\|$. If the lemma fails, then there is a subspace $W$ in $Y$ so that $\operatorname{dim}(T W)^{\perp}>\operatorname{dim} W^{\perp}$. By lemma 5 , there is an element $x^{*} \in S_{(T W)^{\perp}}$ with $d\left(x^{*}, W^{\perp}\right)=1=\sup \left\{x^{*}(x): x \in B_{W}\right\}$. Now, for any $\delta>0$, there is an $x \in B_{W}$ so that $x^{*}(x) \geq 1-\delta$. Since $x^{*}(T(W))=0$, we have,

$$
1-\delta \leq x^{*}(x)=x^{*}(x-T x) \leq \lambda\left\|x^{*}\right\|\|x\|=\lambda .
$$

But, this is a contradiction for $1-\delta>\lambda$. Therefore,

$$
\operatorname{codim}_{X} W=\operatorname{dim} W^{\perp} \geq \operatorname{dim}(T W)^{\perp}=\operatorname{codim}_{X} T(W) .
$$


Lemma 7. Let $Y$ be a subspace of a Banach space $X$ and $T: Y \rightarrow X$ be a linear operator with $\left\|\left.(I-T)\right|_{Y}\right\|<\frac{1}{2}$. Then, $\left\|\left.\left(I-T^{-1}\right)\right|_{T Y}\right\|<1$.

Proof. For any $x \in X$,

$$
\|T x\| \geq\|x\|-\|(I-T) x\| \geq \frac{1}{2}\|x\| .
$$

It follows that $\left\|\left.T^{-1}\right|_{T Y}\right\| \leq 2$. Now, for every $y \in T Y$,

$$
\left\|\left(I-T^{-1}\right) y\right\|=\left\|(I-T) T^{-1} y\right\| \leq\|I-T\|\left\|T^{-1} y\right\| \leq\|I-T\| 2\|y\| .
$$

We can now prove the main result of this section. Again, the proof is inspired by Hilding [6].

Theorem 8. Let $Y$ be a subspace of a Banach space $X, \lambda_{1}, \lambda_{2} \in[0,1)$, and $T$ : $Y \rightarrow X$ a linear operator satisfying, for all $x \in Y$,

$$
\|(I-T) x\| \leq \lambda_{1}\|x\|+\lambda_{2}\|T x\| .
$$

Then $\operatorname{codim}_{X} Y=\operatorname{codim}_{X} T(Y)$.

Proof. To simplify the proof, let $\lambda=\max \left\{\lambda_{1}, \lambda_{2}\right\}$. With $T_{\alpha}$ defined as in Proposition 1 (3), we have from our Basic Inequality, for all $0 \leq \alpha \leq 1$,

$$
\frac{1-\lambda}{1+\lambda}\|x\| \leq\left\|T_{\alpha} x\right\|
$$

Next, we let

$$
E=\left\{0 \leq \alpha \leq 1: \operatorname{codim}_{X} T_{\alpha} Y=\operatorname{codim}_{X} Y\right\}
$$

If $\alpha=0$, then $T_{\alpha}=I$, so $0 \in E \neq \phi$.

Next, we will show that for all $\alpha$ sufficiently close to $\beta$, we have $\operatorname{codim}_{X} T_{\alpha} Y=$ $\operatorname{codim}_{X} T_{\beta} Y$. To see this, given $0 \leq \alpha, \beta \leq 1$, we have

$$
\left\|T_{\alpha} x-T_{\beta} x\right\|=\|(\alpha-\beta) T x-(\alpha-\beta) x\| \leq|\alpha-\beta|(1+\|T\|)\|x\| .
$$

Hence if we let

$$
\epsilon=\frac{1}{2} \frac{1-\lambda}{1+\lambda} \frac{1}{1+\|T\|}
$$

then as long as $|\alpha-\beta| \leq \epsilon$, and applying (7) we have

$$
\left\|T_{\alpha} x-T_{\beta} x\right\| \leq \frac{1}{2\left\|T_{\alpha}^{-1}\right\|}\|x\| .
$$


Now define an operator $L: T_{\alpha}(X) \rightarrow T_{\beta}(X)$ by $L T_{\alpha} x=T_{\beta} x$. By inequality (8), we have that $\|I-L\|<\frac{1}{2}$. Thus by lemma 7 , we can apply lemma 6 to both $L$ and $L^{-1}$ to conclude that $\operatorname{codim}_{X} T_{\alpha} Y=\operatorname{codim}_{X} T_{\beta} Y$.

Summarizing, we have that $0 \in E$, and whenever $\alpha \in E$, we have that $(\alpha-\epsilon, \alpha+$ $\epsilon) \cap[0,1] \subset E$. Hence, $E=[0,1]$ and so $1 \in E$, which is what we needed.

Theorem 8 gives a generalization of the result of Neumann [13].

Corollary 9. If $Y, Z$ are subspaces of a Banach space $X$, and if $T: Y \rightarrow Z$ is a surjective linear operator with $\left\|\left.(I-T)\right|_{Y}\right\|<1$, then $\operatorname{codim}_{X} Y=\operatorname{codim}_{X} Z$.

We could obtain Corollary 9 directly from the Neumann series if $T$ had an extension $\hat{T}$ to all of $X$ also satisfying $\|I-\hat{T}\|<1$. In fact, we can get such an extension if there is a projection $P: X \rightarrow Y$ with $\|P\|<1$ (or just $\|P\|<\|I-T\|$ ). In this case we define $\hat{T}: X \rightarrow X$ by $\hat{T} x=T P x+(I-P) x$. Now, for all $x \in X$,

$$
\|(I-\hat{T}) x\|=\|(I-T) P x\| \leq\|I-T\|\|P x\| \leq\|I-T\|\|x\| .
$$

However, in general $T$ need not have an extension which is an isomorphism on $X$. Our next example shows that even if $\operatorname{dim} Y<\infty$, there need not be an extension $\hat{T}$ of $T$ satisfying $\|I-\hat{T}\|<1$.

Example 10. Let $X=\ell_{p} \oplus \ell_{p}$, for $p \neq 2$. Choose a subspace $W$ in $\ell_{p}$ which is isomorphic to $\ell_{p}$ but uncomplemented in $\ell_{p}$. Let $Y=W \oplus 0$, and let $\left\{\left(f_{n}, 0\right)\right\}$ be the unit vector basis of $\ell_{p}$ in $Y$, and $\left\{e_{n}\right\}$ be the unit vector basis of $\ell_{p}$. Also, let

$$
Z=\left\{\left(\sum a_{n} f_{n}, \frac{1}{K} \sum a_{n} e_{n}\right):\left(a_{n}\right) \in \ell_{p}\right\},
$$

where $K$ is chosen so that,

$$
\frac{1}{K}\left\|\sum a_{n} e_{n}\right\| \leq \frac{1}{2}\left\|\sum a_{n} f_{n}\right\| .
$$

Finally, define $T: Y \rightarrow Z$ by

$$
T\left(\sum a_{n} f_{n}, 0\right)=\left(\sum a_{n} f_{n}, \frac{1}{K} \sum a_{n} e_{n}\right) .
$$

Then,

(1) $\|I-T\|<1$,

(2) $Z$ is complemented in $X$,

(3) $T$ is an isomorphism of $Y$ onto $Z$. 
Therefore, $T$ cannot be extended to be an isomorphism of $X$ onto $X$.

Proof. (1) We just apply (9) to get

$$
\left\|(I-T)\left(\sum a_{n}\left(f_{n}, 0\right)\right)\right\|_{X}=\frac{1}{K}\left\|\sum a_{n} e_{n}\right\|_{\ell_{p}} \leq \frac{1}{2}\left\|\sum a_{n} f_{n}\right\| .
$$

(2) we define the operator $P$ on $X$ by:

$$
P\left(\sum a_{n} e_{n}, \sum b_{n} e_{n}\right)=\left(K \sum b_{n} f_{n}, \sum b_{n} e_{n}\right) .
$$

It is clear that $P$ is a bounded linear projection of $X$ onto $Z$.

(3) This is clear since the operator $T\left(f_{n}\right)=\left(f_{n}, e_{n}\right)$ is an isomorphism.

By a standard compactness arguement, we can finite dimensionalize the above example. There is a choice of natural numbers $j_{1}<j_{2}<j_{3}<\cdots$ with the following property. Let $Y_{n}=\operatorname{span}_{1 \leq i \leq j_{n}}\left(f_{i}, 0\right)$ and $Z_{n}=\operatorname{span}_{1 \leq i \leq j_{n}}\left(f_{i}, \frac{1}{K} e_{i}\right)$, and $T_{n}=\left.T\right|_{Y_{n}}$. Then there is a $0<\lambda<1$ so that for all $n=1,2,3, \ldots,\left\|I-T_{n}\right\|<\lambda$, but for any extension $\hat{T}_{n}$ of $T_{n}$ to all of $X$, we have $\left\|\hat{T}_{n}\right\|\left\|\hat{T}_{n}^{-1}\right\| \geq n$.

The next corollary of Theorem 8 comes from mimmicking the proof of lemma 4 .

Corollary 11. If $X, Y$ are Banach spaces and $S, T: X \rightarrow Y$ are linear operators satisfying for all $x \in X$,

$$
\|S x-T x\| \leq \lambda(\|S x\|+\|T x\|),
$$

then $\operatorname{codim}_{Y}(\overline{S(X)})=\operatorname{codim}_{Y}(\overline{T(X)})$.

Given an operator $T: X \rightarrow Y$ with closed range, we let

$$
\alpha(T)=\operatorname{dim} \operatorname{ker} T
$$

and

$$
\beta(T)=\operatorname{dim} Y / T(X) .
$$

If either $\alpha(T)<\infty$ or $\beta(T)<\infty$ we define the Fredholm index i(T) of $T$ by $i(T)=\alpha(T)-\beta(T)$. If $\alpha(T)$ and $\beta(T)$ are both finite (i.e. if $i(T)$ is defined and is finite) then $T$ is called a Fredholm operator of index $i(T)$.

Corollary 12. Let $X, Y$ be Banach spaces and $S, T: X \rightarrow Y$ be linear operators satisfying for all $x \in X$, and fixed $0 \leq \lambda_{1}, \lambda_{2}<1$,

$$
\|(S-T) x\| \leq \lambda_{1}\|S x\|+\lambda_{2}\|T x\| .
$$

If $S$ is a Fredholm operator with Fredholm Index $n$, then $T$ is also a Fredholm operator with Fredholm Index $n$.

Proof. By our Basic Inequality, ker $S=\operatorname{ker} T$. Now apply Theorem 8 .

We end with one final application of Theorem 8. 
Corollary 13. Let $X$ be a Banach space and $T: X \rightarrow X$ be a linear operator satisfying

$$
\|(I-T) x\| \leq \lambda(\|x\|+\|T x\|)
$$

for all $x \in X$, and fixed $0 \leq \lambda<1$. Then, for all natural numbers $n$, and all $x \in X$, we have, $x \in \overline{\operatorname{span}_{n \leq k} T^{k} x}$.

Proof. For each $n=0,1,2, \cdots$, let $W_{n}=\overline{\operatorname{span}_{n \leq k} T^{k} x}$. Then $T$ maps $W_{n}$ into $W_{n}$ and satisfies (10), and hence is onto. Since $T\left(W_{n}\right)=W_{n+1}$, we see that $W_{0}=W_{1}=W_{2}=\cdots$, which proves the corollary.

ACKNOWLEDGEMENT. The authors thank Ole Christensen for helpful discussions concerning this paper.

\section{REFERENCES}

1. M.G. Arsove, The Paley-Wiener theorem in metric linear spaces, Pacific J. Math 10 (1960), 365-379.

2. Y. Benyamini and Y. Sternfeld, Spheres in infinite-dimensional normed spaces are Lipschitz contractible, Proceedings of the American Mathematical Society 88 no. 3 (1983), 439-445.

3. R.P. Boas, Jr., Expansions of analytic functions, Transactions of the American Mathematical Society 48 (1940), 467-487.

4. M.M. Day, On the basis problem in normed spaces, Proceedings of the American Mathematical Society 13 (1962), 655-658.

5. I.C. Gokhberg and M.G. Krein, Fundamental theorems on deficiency numbers, root numbers, and indices of linear operators (Russian), Usp. Mat. Nauk 12 (1957.), 43-118. Translated in American Mathematical Society Translations, ser. 2, vol. 13.

6. S.H. Hilding, Note on completeness theorems of Paley-Wiener type, Annals of Math 49 (1948), 953-955.

7. N.J. Kalton, N.T. Peck, and J.W. Roberts, An F-space sampler, London Mathematical Society Lecture Notes, Cambridge University Press 89 (1984).

8. M.G. Krein, M.A. Krasnoselskii, and D.P. Milman, On the defect numbers of linear operators in a Banach space and on some geometrical questions (Russian), Sb. Tr. Inst. Mat. Akad. Nauk Ukr. SSR 11 (1948), 97-112.

9. B. Sz. Nagy, Expansion theorems of Paley-Wiener type, Duke Math. Journal 14 (1947), 975978.

10. R.E.A.C. Paley and N. Wiener, Fourier transforms in the complex domain, New York (1934).

11. H. Pollard, Completeness theorems of Paley-Wiener type, Annals of Math 45 (1944), 738-739.

12. James R. Retherford, Basic sequences and the Paley-Wiener criterion, Pacific Journal of Math 14 no. 3 (1964), 1019-1027.

13. C. Von Newmann, Untersuchungen uber das logarithmische und Newtonsche Potential, Teubner, Leipzig (1877).

Department of Mathematics, The University of Missouri, Columbia, Missouri 65211, USA

E-mail address: pete@casazza.math.missouri.edu; nigel@math.missouri.edu 